\title{
SPECTRA OF CONJUGATED IDEALS IN GROUP ALGEBRAS OF ABELIAN GROUPS OF FINITE RANK AND CONTROL THEOREMS
}

\author{
by ANATOLII V. TUSHEV
}

(Received 15 March, 1995; revised 22 August, 1995)

1. Introduction. Throughout $k$ will denote a field. If a group $\Gamma$ acts on a set $A$ we say an element is $\Gamma$-orbital if its orbit is finite and write $\Delta_{\Gamma}(A)$ for the subset of such elements. Let $I$ be an ideal of a group algebra $k A$; we denote by $I^{+}$the normal subgroup $(I+1) \cap A$ of $A$. A subgroup $B$ of an abelian torsion-free group $A$ is said to be dense in $A$ if $A / B$ is a torsion-group. Let $I$ be an ideal of a commutative ring $K$; then the spectrum $\mathrm{Sp}(I)$ of $I$ is the set of all prime ideals $P$ of $K$ such that $I \leq P$. If $R$ is a ring, $M$ is an $R$-module and $x \in M$ we denote by $\mathscr{A}_{R}(x)$ the annihilator of $x$ in $R$. We recall that a group $\Gamma$ is said to have finite torsion-free rank if it has a finite series in which each factor is either infinite cyclic or locally finite; its torsion-free rank $r_{0}(\Gamma)$ is then defined to be the number of infinite cyclic factors in such a series.

Let $A$ be an abelian torsion-free group of finite rank acted upon by a group $\Gamma$ and let $I$ be an ideal of $k A$. The subgroup $S_{\Gamma}(A)$ of $\Gamma$ of elements $\gamma$ such that $I \cap k B=I^{\gamma} \cap k B$ for some finitely generated dense subgroup $B$ of $A$ is said to be the standardiser of $I$. We will say that an ideal $I$ of $k A$ is locally prime if $I \cap k B$ is a prime ideal of $k B$ for some dense finitely generated subgroup $B$ of $A$. It easily follows from Wilson's version [18, Section 3.11] of an important theorem of Brookes [1, Theorem A] that if $\Delta_{\Gamma}(A)=1, I$ is a locally prime ideal of $k A$ and $S_{\Gamma}(I)=\Gamma$, then $I^{+} \neq 1$. But, of course, $I^{+}$may contain no non-trivial $\Gamma$-invariant subgroup.

Let $G$ be a group with a torsion-free abelian normal subgroup $A$ of finite rank. In [12, Theorem E] Nabney proved that if $M$ is any $k G$-module which is not $k A$-torsion-free then there is an element $a \in M \backslash\{0\}$ such that $a k G=(a k S) \otimes_{k S} k G$, where $S=S_{G}(P)$ for some $P \in \operatorname{Sp}\left(\mathscr{A}_{k A}(a)\right)$. But, generally, if $G$ has finite torsion-free rank then $r_{0}\left(S / C_{s}(a k S)\right)$ may be the same as $r_{0}(G)$ for any $a \in M \backslash\{0\}$. However, it would be very useful to find such a subgroup $H$ of $G$ that $a k G=(a k H) \otimes_{k H} k G$ and $r_{0}\left(H / C_{H}(a k H)\right)<r_{0}(G)$ for some $a \in M \backslash\{0\}$, because it would be possible to use induction on $r_{0}(G)$ for the study of $M$ then. The search for such a subgroup $H$ is the main aim of this paper. In the case of a polycyclic group $G$ this approach was applied by Roseblade in [14].

Let $A$ be an abelian torsion-free group of finite rank acted upon by a group $\Gamma$ and let $I$ be an ideal of $k A$. We say that a subgroup $\Lambda$ of $S_{\Gamma}(I)$ separates $I$ if $\operatorname{Sp}(I) \cap \operatorname{Sp}\left(I^{\gamma}\right)=\varnothing$ for any $\gamma \in S_{\Gamma}(I)$ which is not contained in $\Lambda$. It is not difficult to note, that the intersection $\operatorname{Sep}_{\Gamma}(I)$ of all subgroups separating $I$ also separates $I$; $\operatorname{Sep}_{\Gamma}(I)$ will be called the separator of $I$. Evidently, $\operatorname{Sep}_{\Gamma}(I) \leq S_{\Gamma}(I)$. We prove that if $k$ is a field of characteristic zero, $\Gamma$ is a soluble group of finite torsion-free rank and $M$ is a $k A$-module such that $\mathscr{A}_{k A}(x)$ is a non-zero locally prime ideal of $k A$ and $r_{0}(\Gamma)=r_{0}\left(\operatorname{Sep}_{\Gamma}\left(\mathscr{A}_{k A}(x)\right)\right.$ for some element $x \in M \backslash\{0\}$ then there is an element $y \in M \backslash\{0\}$ such that $\mathscr{A}_{k A}^{+}(y)$ has a non-trivial $\operatorname{Sep}_{\mathrm{r}}\left(\mathscr{A}_{k A}(y)\right.$ )-invariant subgroup (Theorem 3.8). This theorem allows us to obtain our main result-a control theorem for modules over group algebras of soluble gorups of finite torsion-free rank (which will be our Theorem 4.2).

Glasgow Math. J. 38 (1996) 309-320. 
THEOREM. Let $G$ be a soluble group of finite torsion-free rank and let $A$ be $a$ torsion-free abelian normal subgroup of $G$ such that $\Delta_{G}(A)=1$. Let $k$ be a field of characteristic zero and let $M$ be a $k G$-module. If $M$ is not $k A$-torsion-free then there is an element $a \in M \backslash\{0\}$ such that $a k G=(a k H) \otimes_{k H} k G$ and $r_{0}\left(H / C_{H}(a k H)\right)<r_{0}(G)$, where $H=\operatorname{Sep}_{G}\left(\mathscr{A}_{k A}(a)\right)$.

We should note that some other approaches to control theorems for modules over group rings of infinite groups were developed by Brookes and Brown (see [2] and [3]).

We recall that a group $G$ has finite Prüfer rank if there is an integer $r$ such that each finitely generated subgroup of $G$ can be generated by $r$ elements; its Pruifer rank $r(G)$ is then the least integer $r$ with this property. As an application of Theorem 4.2, we consider faithful irreducible representations of a finitely generated metabelian group $G$ of finite Prüfer rank over a field $k$ of characteristic zero. We prove that if $G$ is not nilpotent-by-finite then each such representation is induced from an irreducible representation of a subgroup $H \leq G$ such that $r_{0}(H)<r_{0}(G)$ (Theorem 5.5). If $G$ is an abelian-by-cyclic group it implies that any faithful irreducible representation of $G$ over $k$ is induced from an irreducible representation of an abelian subgroup of $G$ (Corollary 5.6). Irreducible representations of some abelian-by-cyclic groups were considered by Musson in [10]. Irreducible representations of finitely generated nilpotent groups were considered by Harper [7] and by Segal [15], and irreducible representations of polycyclic groups were considered by Harper [8] and by Musson [11].

By [6], any finitely generated metabelian group of finite Prüfer rank is a minimax abelian-by-polycyclic group. A minimax group is a group with a finite series each of whose factors satisfies either the minimal condition or the maximal condition for subgroups. Irreducible representations of minimax abelian-by-polycyclic groups under certain additional conditions where considered by Nabney [12].

2. Some properties of Cernikov modules. This section is auxiliary; its main result (Proposition 2.6) will be used in the proof of Theorem 3.5.

Let $R$ be a ring. An $R$-module $A$ is said to be cyclic if it is generated by one element. By the $\operatorname{socle} \operatorname{Soc}(A)$ of an $R$-module $A$ we mean the submodule of $A$ which is generated by the minimal submodules of $A$; if $A$ has no minimal submodule then $\operatorname{Soc}(A)=0$.

Lemma 2.1. Let $A$ be a $\mathbb{F}_{p}[g]$-module. Then the module $A$ is cyclic if and only if $\operatorname{Soc}(A)$ is cyclic.

Proof. This assertion holds because $\mathbb{F}_{p}[g]$ is a principal ideals domain.

Let $R$ be a ring. An $R$-module $A$ is said to be Cernikov if its additive group is Črnikov, that is, a direct sum of finitely many cyclic and quasi-cyclic groups (see [9]). If the additive group of $A$ is a $p$-group then $\Omega_{n}(A)$ is the submodule of $A$ which consists of all elements $x \in A$ such that $x p^{n}=0$, where $n \in \mathbb{N}$.

Let $R$ be a ring. An infinite $R$-module $A$ is said to be minimal infinite (or m.i.-module) if any proper submodule of $A$ is finite. It is not difficult to show that if $A$ is a Cernikov m.i.-module then $A$ is a divisible $p$-group. 
Lemma 2.2. Let $A$ be a Cernikov $\mathbb{Z}[g]$-module. Suppose that the additive group of $A$ is a $p$-group and the socle of $A$ is cyclic. Then for any m.i.-submodule $B$ of $A$ the socle of the quotient module $A / B$ is cyclic.

Proof. Obviously, it is sufficient to show that the socle of $\Omega_{1}(A / B)$ is cyclic. Since $B$ is a divisible group, it is not too difficult to show that $\Omega_{1}(A / B)=\left(\Omega_{1}(A)+B\right) / B=$ $\Omega_{1}(A) /\left(\Omega_{1}(A) \cap B\right)$. As $\Omega_{1}(A)$ has cyclic socle, it easily follows from Lemma 2.1 that the quotient module $\Omega_{1}(A / B) \simeq \Omega_{1}(A) /\left(\Omega_{1}(A) \cap B\right)$ has cyclic socle.

LEMMA 2.3. Let $A$ be a Cernikov $\mathbb{Z}[g]-m o d u l e$ and let $A$ be the group of $\mathbb{Z}[g]$-automorphisms of $A$. Suppose that $A$ is a divisible $p$-group. Then:

(i) if $\operatorname{Soc}(A)$ is cyclic then $\mathscr{A}$ is abelian;

(ii) for any finite submodule $X$ of $A \oplus A$ the socle of the quotient module $(A \oplus A) / X$ is not cyclic.

Proof. (i) Since $\operatorname{Soc}(A)$ is cyclic and, evidently, $\operatorname{Soc}(A)=\operatorname{Soc}\left(\Omega_{1}(A)\right)$, by Lemma 2.1, $\Omega_{1}(A)$ is cyclic. It easily follows that $\Omega_{n}(A)$ is cyclic for each $n \in \mathbb{N}$. Then $\Omega_{n}(A)=K_{n}=\mathbb{Z}[g] / I_{n}$ for each $n \in \mathbb{N}$, where $I_{n}$ is an ideal of $\mathbb{Z}[g]$. Let $\mathscr{A}_{n}$ be the group of $\mathbb{Z}[g]$-automorphisms of $\Omega_{n}(A)$; it is well known that $\mathscr{A}_{n} \approx U\left(K_{n}\right)$, where $U\left(K_{n}\right)$ is the group of units of $K_{n}$, and hence $\mathscr{A}_{n}$ is abelian. As $\mathscr{A} / C_{\mathscr{A}}\left(\Omega_{n}(A)\right) \leq \mathscr{A}_{n}$ and $\cap_{n \in \mathbb{N}}$ $C_{\mathscr{A}}\left(\Omega_{n}(A)\right)=1$, it follows that $\mathscr{A}$ is abelian.

(ii) Suppose that for some finite submodule $X$ of $B=A \oplus A$ the socle of $B / X$ is cyclic. Let $\mathscr{A}$ be the group of $\mathbb{Z}[g]$-automorphisms of $B$ and $\mathscr{B}$ be the group of $\mathbb{Z}[g]$-automorphisms of $B / X$. Then, by (i), $\mathscr{B}$ is abelian. Let $\mathcal{N}$ be the normalizer of $X$ in $\mathscr{A}$ then, as $X \leq \Omega_{n}(B)$ for some $n \in \mathbb{N}$, it is not difficult to show that $|\mathscr{A}: \mathcal{N}|<\infty$. As each $v \in \mathcal{N}$ induces a $\mathbb{Z}[g]$-automorphism of $B / X$, there is a homomorphism $\varphi: \mathcal{N} \rightarrow \mathscr{B}$ such that $\operatorname{ker} \varphi=C_{\mathcal{N}}(B / X)$. Let $\alpha \in \operatorname{ker} \varphi$; then $B(1-\alpha) \leq X$ and hence, as $X$ is finite, there is $n \in \mathbb{N}$ such that $B p^{n}(1-\alpha)=0$. Therefore, as the additive group of $A$ is divisible, $B(1-\alpha)=0$ and hence $\alpha=1$. So, $\mathcal{N} \leq \mathscr{B}$ because $\operatorname{ker} \varphi=1$. Thus $\mathcal{N}$ is an abelian group and hence $\mathscr{A}$ is an almost abelian group.

On the other hand, evidently, $\mathscr{A}$ contains the linear group $G L_{2}(\mathbb{Z})$ and it is well known that $G L_{2}(\mathbb{Z})$ is not almost abelian. This is a contradiction.

Lemma 2.4. Let $A$ be a Cernikov $\mathbb{Z}[g]$-module and let $\mathcal{M}$ be the set of all m.i.-submodules of $A$. If the socle of $A$ is cyclic then $\mathcal{M}$ is finite.

Proof. As any submodule of $A$ is the direct sum of its Sylow components, any m.i.-submodule of $A$ is contained in some Sylow component of $A$. Thus we may assume that the additive group of $A$ is a $p$-group.

The proof is by induction on Prüfer rank of the additive group of $A$. Let $B$ be an m.i.-submodule of $A$. Then, by Lemma $2.2, \operatorname{Soc}(A / B)$ is a cyclic $\mathbb{Z}[g]$-module and hence, by the induction hypothesis, the set of all m.i.-submodules of $A / B$ is finite. Thus it is sufficient to consider the case when $A / B$ is an m.i.-module.

Suppose that $\mathcal{M}$ is infinite and let $A_{i} \in \mathcal{M}, A_{i} \neq B$, where $i=1,2$. Put $X_{i}=A_{i} \cap B$; then $\left|X_{i}\right|<\infty$ and

$$
A_{i} / X_{i}=\left(A_{i}+B\right) / B=A / B
$$

where $i=1,2$. Put $X=X_{1}+X_{2}, \hat{A}=A / X$ and $\hat{A}_{i}=\left(A_{i}+X\right) / X$; then, as $A_{i} \cap X=X_{i}$, by 
(1), $\hat{A}_{1} \simeq \hat{A}_{2}$. Evidently, $\hat{A}=\hat{A}_{1}+\hat{A}_{2}$; then it is not difficult to show that there is a finite submodule $Y \leq \hat{A}_{1} \oplus \hat{A}_{2}$ such that

$$
\hat{A} \simeq \hat{A}_{1} \oplus \hat{A}_{2} / Y
$$

On the other hand, there is $n \in \mathbb{N}$ such that $X \leq \Omega_{n}(A)$ and hence, as $A \simeq A / \Omega_{n}(A)$, there is a finite submodule $Z \leq \hat{A}$ such that $\hat{A} / Z \simeq A$. Then, by (2), $\left(\hat{A}_{1} \oplus \hat{A}_{2}\right) / D \simeq A$ for some finite submodule $D \leq \hat{A}_{1} \oplus \hat{A}_{2}$ but this contradicts Lemma 2.3 (ii).

Lemma 2.5. Let $A$ be a Cernikov $\mathbb{Z}[g]$-module and let $k$ be a field of characteristic zero. Let $M$ be a $k A$-module, $x \in M$ and $P \in \mathrm{Sp}\left(\mathscr{A}_{k A}(x)\right)$. Then;

(i) for any finite subgroup $B \leq A$ there is an element $y \in M$ such that $\mathscr{A}_{k A}(y) \cap k B=$ $P \cap k B=D$ is a maximal ideal of $k B$ and $P \geq \mathscr{A}_{k A}(y)$;

(ii) if $A^{\prime}$ is an m.i.-submodule of $A$ and $P^{+}$does not contain $A^{\prime}$ then there is an element $y \in M$ such that for any $L \in \operatorname{Sp}\left(\mathscr{A}_{k A}(y)\right) L^{+}$does not contain $A^{\prime}$.

Proof. (i) Put $D=P \cap k B$ then $D \in \mathrm{Sp}\left(\mathscr{A}_{k B}(x)\right)$. By Maschke's theorem, $T=x k B$ is a semisimple $k B$-module. Then there is a simple submodule $S \leq T$ which is annihilated by $D$. Thus $y$ may be chosen as a non-zero element of $S$. Evidently, $\operatorname{Sp}\left(\mathscr{A}_{k A}(y)\right)$ consists of all $L \in \mathrm{Sp}\left(\mathscr{A}_{k A}(x)\right)$ such that $L \cap k B=D$ and hence $P \geq \mathscr{A}_{k A}(y)$.

(ii) Evidently, there is a finite subgroup $B \leq A^{\prime}$ which is not contained in $P^{+}$. By (i), there is an element $y \in M$ such that $\mathscr{A}_{k A}(y) \cap k B=P \cap k B=D$. As $D$ is a maximal ideal of $k B, P \cap k B=D$ for any $L \in \mathrm{Sp}\left(\mathscr{A}_{k A}(y)\right)$. Therefore, for any $L \in \mathrm{Sp}\left(\mathscr{A}_{k A}(y)\right), L^{+}$does not contain $B$ and hence $L^{+}$does not contain $A^{\prime}$.

Proposition 2.6. Let $A=\bigoplus_{i=1}^{n} A_{i}$ be a Cernikov $\mathbb{Z}[\mathrm{g}]-$ module such that $\operatorname{Soc}\left(A_{i}\right)$ is cyclic for each $i$. Let $k$ be a field of characteristic zero, and let $M$ be a $k A$-module. Then there is an element $a \in M \backslash\{0\}$ such that for any $x \in a k A$ and, for each $1 \leq i \leq n$, $k C_{i} \cap \mathscr{A}_{k A}(x)=P_{i}$ is a maximal ideal of $k C_{i}$, where $C_{i} / H_{i}=\operatorname{Soc}\left(A_{i} / H_{i}\right)$ and $H_{i}$ is the maximal g-invariant subgroup of $\mathscr{A}_{k A}^{+}(x) \cap A_{i}$.

Proof. The proof is by induction on $n$.

Consider first the case where $n=1$. The proof is by induction on Prüfer rank of the additive group of $A$. Suppose that there is an element $x \in M \backslash\{0\}$ such that $\mathscr{A}_{k A}^{+}(x)$ has an m.i.-submodule $A^{\prime}$. Then $x k A$ may be considered as a $k\left(A / A^{\prime}\right)$-module and, by Lemma 2.2 , we may use the induction hypothesis. Thus we may assume that $\mathscr{A}_{k A}^{+}(x)$ contains no m.i.-submodule for any $x \in M \backslash\{0\}$.

Let $\mathcal{M}=\left\{A_{1}, \ldots, A_{m}\right\}$ be the set of all m.i.-submodules of $A$; by Lemma $2.4, \mathcal{M}$ is finite. We will show by induction on $m$ that there is an element $y \in M$ such that for any $P \in \operatorname{Sp}\left(\mathscr{A}_{k A}(y)\right) P^{+}$contains no m.i.-submodule. Suppose that there is $x \in M$ such that for any $P \in \operatorname{Sp}\left(\mathscr{A}_{k A}(x)\right) P^{+}$does not contain submodules $A_{1}, \ldots, A_{m-1}$. It easily follows from Maschke's theorem that the quotient ring $k A / \mathscr{A}_{k A}(x)$ has no nilpotent element and hence it is semiprime. So, $\mathscr{A}_{k A}(x)$ is the intersection of all $P \in \mathrm{Sp}\left(\mathscr{A}_{k A}(x)\right)$. Then, as $\mathscr{A}_{k A}^{+}(x)$ does not contain $A_{m}$, there is $P \in \mathrm{Sp}\left(\mathscr{A}_{k A}(x)\right)$ such that $P^{+}$does not contain $A_{m}$. Therefore, by Lemma 2.5(ii), there is an element $y \in x k A$ such that for any $P \in \operatorname{Sp}\left(\mathscr{A}_{k A}(y)\right) P^{+}$does not contain $A_{m}$. As $y \in x k A, \mathscr{A}_{k A}(x) \leq \mathscr{A}_{k A}(y)$. Therefore, $\operatorname{Sp}\left(\mathscr{A}_{k A}(y)\right) \subseteq \operatorname{Sp}\left(\mathscr{A}_{k A}(x)\right)$ and hence $P^{+}$contains no m.i.submodule for any $P \in \operatorname{Sp}\left(\mathscr{A}_{k A}(y)\right)$. Evidently, $y \neq 0$.

Let $P \in \operatorname{Sp}\left(\mathscr{A}_{k A}(y)\right)$ and let $H$ be the maximal $g$-invariant subgroup of $P^{+}$. As $P^{+}$ 
contains no m.i.-submodule, $H$ is finite. Put $C / H=\operatorname{Soc}(A / H)$ then $|C|<\infty$ and hence, by Lemma 2.5(i), there is an element $a \in M \backslash\{0\}$ such that $\mathscr{A}_{k A}(a) \cap k C=P \cap k C$ and, as $P \geq \mathscr{A}_{k A}(a), H$ is the maximal $g$-invariant subgroup of $\mathscr{A}_{k A}(a)$. Let $x \in a k A$; then $\mathscr{A}_{k A}(x) \cap k C \geq \mathscr{A}_{k A}(a) \cap k C=D$ and, as $D$ is a maximal ideal of $k C, \mathscr{A}_{k A}(x) \cap k C=D$. Let $X$ be the maximal $g$-invariant subgroup of $\mathscr{A}_{k A}(x)$; then, as $\mathscr{A}_{k A}(x) \geq \mathscr{A}_{k A}(a), X \geq H$. Suppose that $X \neq H$; then, as $C / H$ is the socle of $A / H, L=C \cap X>H$. Evidently, $L \leq D^{+} \leq P^{+}$but this is a contradiction, because $H$ is the maximal $g$-invariant subgroup of $P^{+}$.

Consider now the general case. By the induction hypothesis, there is an element $b \in M \backslash\{0\}$ such that for any element $x \in b K a k C_{i} \cap \mathscr{A}_{k A}(x)=P_{i}$ is a maximal ideal in $k C_{i}$, where $C_{i} / H_{i}=\operatorname{Soc}\left(A_{i} / H_{i}\right), H_{i}$ is the maximal $g$-invariant subgroup of $\mathscr{A}_{k A}^{+}(x) \cap A_{i}$ and $2 \leq i \leq n$. By the same arguments, there is an element $a \in b k A \backslash\{0\}$, such that $k C_{1} \cap$ $\mathscr{A}_{k A}(x)=P_{1}$ is a maximal ideal in $k C_{1}$ for any element $x \in a k A$, where $C_{1} / H_{1}=$ $\operatorname{Soc}\left(A_{1} / H_{1}\right)$ and $H_{1}$ is the maximal $g$-invariant subgroup of $\mathscr{A}_{k A}^{+}(x) \cap A_{1}$. Let $x \in a k A$; then, as $a \in b k A, x \in b k A$. Thus $k C_{i} \cap \mathscr{A}_{k A}(x)=P_{i}$ is a maximal ideal in $k C_{i}$, where $C_{i} / H_{I}=\operatorname{Soc}\left(A_{i} / H_{i}\right), H_{i}$ is the maximal $g$-invariant subgroup of $\mathscr{A}_{k A}^{+}(x) \cap A_{i}$ and $1 \leq i \leq n$. rank.

3. On spectra of conjugated ideals of group algebras of abelian groups of finite

Lemma 3.1. Let $A$ be an abelian group acted upon by a group $\Gamma$, and $B$ be a $\Gamma$-invariant subgroup of $A$. Let $k$ be $a$ field and let $I$ be an ideal of $k A$. Then:

(i) if $\operatorname{Sp}\left(I_{1}^{\gamma}\right) \cap \operatorname{Sp}\left(I_{1}\right)=\varnothing$ then $\operatorname{Sp}\left(I^{\gamma}\right) \cap \operatorname{Sp}(I)=\varnothing$, where $\gamma \in \Gamma$ and $I_{1}=I \cap k B$;

(ii) suppose that $B \leq I^{+}$and let $\Delta \leq I$ be the ideal of $k A$ generated by $1-B$. Put $\hat{I}=I / \Delta$. Then $\operatorname{Sp}\left(\hat{I}^{\gamma}\right) \cap \operatorname{Sp}(\hat{I})=\varnothing$ if $\operatorname{Sp}\left(I^{\gamma}\right) \cap \operatorname{Sp}(I)=\varnothing$, where $\gamma \in \Gamma$.

Proof. (i) Suppose that there is $P \in \mathrm{Sp}\left(I^{\gamma}\right) \cap \mathrm{Sp}(I)$. Then, as $I^{\gamma}=I^{\gamma} \cap k B, P_{1} \in$ $\mathrm{Sp}\left(I_{1}^{\gamma}\right) \cap \mathrm{Sp}\left(I_{1}\right)$, where $P_{1}=P \cap k B$. This is a contradiction.

(ii) Suppose that there is $\hat{P} \in \mathrm{Sp}\left(\hat{I}^{\gamma}\right) \cap \mathrm{Sp}(\hat{I})$; then $P=P / \Delta=\left(P_{1} / \Delta\right)^{\gamma}$, where $P$, $P_{1} \in \mathrm{Sp}(I)$. As $B$ is a $\Gamma$-invariant subgroup of $A,\left(P_{1} / \Delta\right)^{\gamma}=P \gamma / \Delta$ and hence $P=P$. This is a contradiction.

LeмmA 3.2. Let $A$ be an abelian torsion-free group of finite rank acted upon by $a$ soluble group $\Gamma$ such that $C_{\Gamma}(A)=1$. Then:

(i) $\Gamma$ has a torsion-free normal subgroup of finite index;

(ii) if $A \otimes_{\mathbf{Z}} \mathbb{Q}$ is a simple $\mathbb{Q} \Gamma$-module then $\Gamma$ has a free abelian normal subgroup of finite index. [16]).

Proof. These assertions are well known properties of linear soluble groups (see

LEMMA 3.3. Let $F$ be a finitely generated abelian group and let $R$ be a prime ideal of $\mathbb{Z} F$ such that $K=\mathbb{Z} F / R$ is a torsion-free group of finite rank. Let $J$ be a dense subgroup of $K$. Then there are $a \mathbb{Z} F$-endomorphism $g$ of $K$ and $a \mathbb{Z}[g]$-submodule $H$ of $J$ such that the quotient module $K / H$ is Cernikov and $\operatorname{Soc}(K / H)$ is cyclic.

Proof. Let $\hat{R}$ be the field of fractions of $K$. Since $\hat{R}$ is finite-dimensional over $\mathbb{Q}$, it is well known that there is an algebraic integer $\xi \in \hat{R}$ such that $\hat{R}=\mathbb{Q}(\xi)$. As $\xi$ is an 
algebraic integer, there is $n \in \mathbb{N}$, such that any element $b \in \mathbb{Z}[\xi]$ may be written in the form $b=a_{0}+a_{1} \xi+\ldots+a_{n} \xi^{n}$, where $a_{i} \in \mathbb{Z}$. Since $K$ is a dense subgroup of $\hat{R}$ and $J$ is a dense subgroup of $K, J$ is a dense subgroup of $\hat{K}$ and hence for any $\xi^{i}$ there is $m_{i} \in \mathbb{N}$, such that $\xi^{i} m_{i} \in J$. Then $\mathbb{Z}[\xi] m \leq J$, where $m=\prod_{i=0}^{n} m_{i}$. Put $g=\xi m$; then $m \mathbb{Z}[g] \leq$ $\mathbb{Z}[\xi] m \leq J$. As $g \in K$ and $K$ is a ring, $g$ can be considered as a $\mathbb{Z} F$-endomorphism of $K$ and $K$ can be considered as a $\mathbb{Z}[g]$-module. Put $H=m \mathbb{Z}[g]$. Evidently, $\widehat{K}=\mathbb{Q}(g)$ and hence $H$ is a dense subgroup of $K$ and, as, by [6, Lemma 5.1], the group $K$ is minimax, the quotient module $K / H$ is Cernikov. We now show that $\operatorname{Soc}(K / H)$ is cyclic. Since $K / H \leq R / H$, it is sufficient to show that $\operatorname{Soc}\left(\hat{K}_{p} / H\right)$ is cyclic for any Sylow $p$-component $\hat{K}_{p} / H$ of the quotient module $\hat{K} / H$. Evidently, $H / H p \simeq \Omega_{1}\left(\hat{R}_{p} / H\right)$. As $H$ is a cyclic $\mathbb{Z}[g]$-module, $H / H p$ is a cyclic $\mathbb{Z}_{p}[g]$-module and, as $H / H p \simeq \Omega_{1}\left(\widehat{K}_{p} / H\right)$, by Lemma 2.1 , $\operatorname{Soc}\left(\Omega_{1}\left(\widehat{K}_{p} / H\right)\right)$ is cyclic.

LEMMA 3.4. Let $A$ be an abelian group acted upon by a group $\Gamma$ let $k$ be a field and let $I$ be an ideal of $k A$. Let $L$ be a subgroup of $A$ such that $I^{+}$does not contain $L$ and suppose that $P=k L \cap I$ is a maximal ideal of $k L$. If $\gamma \in \Gamma$ and $L \leq\left(I^{+}\right)^{\gamma}$ then $\operatorname{Sp}\left(I^{\gamma}\right) \cap \operatorname{Sp}(I)=\varnothing$.

Proof. Evidently, $L \leq\left(I^{\gamma}\right)^{+}$and hence $k L \cap I^{\gamma}=\Delta=\langle h-1 \mid h \in L\rangle$. Then, as $\Delta$ is a maximal ideal of $k I, \operatorname{Sp}\left(k L \cap I^{\gamma}\right)=\{\Delta\}$. Suppose that $\mathrm{Sp}\left(I^{\gamma}\right) \cap \mathrm{Sp}(I) \neq \varnothing$; then $\mathrm{Sp}\left(I^{\gamma} \cap\right.$ $k L) \cap \operatorname{Sp}(I \cap k L) \neq \varnothing$ and hence, as $\operatorname{Sp}(I \cap k L)=\{P\}, \Delta=P$. Then, as $P \leq I, \Delta \leq I$ and hence $L \leq I^{+}$. This is a contradiction.

THEOREM 3.5. Let $A$ be an abelian torsion-free group of finite rank acted upon by a soluble group $\Gamma$ of finite torsion-free rank and let $k$ be a field of characteristic zero. Let $M$ be a $k A$-module which contains a non-zero element $x$ such that $\mathscr{A}_{k A}^{+}(x)$ is a dense subgroup of $A$. Then there is an element $y \in M \backslash\{0\}$ such that $\mathscr{A}_{k A}^{+}(y)$ has a non-trivial subgroup $W$ such that $\operatorname{Sp}\left(\mathscr{A}_{k A}(y)\right) \cap \operatorname{Sp}\left(\mathscr{A}_{k_{A}}(y)\right)=\varnothing$ if $\gamma \in \Gamma$ and $\gamma$ is not contained in $N_{\Gamma}(W)$, where $N_{\Gamma}(W)$ is the normalizer of $W$ in $\Gamma$.

Proof. By Lemma 3.1(i), in the proof $A$ may be changed to any of its proper $\Gamma$-invariant subgroups. So, we can assume that $A$ is a $\mathbb{Z} \Gamma$-module generated by one element $z \in A$ and $A \otimes_{\mathbb{Z}} \mathbb{Q}$ is a simple $\mathbb{Q} \Gamma$-module. We can also assume that $C_{\Gamma}(A)=1$. Then, by Lemma 3.2(ii), $\Gamma$ has a finitely generated abelian normal subgroup $F$ of finite index and, as $A$ is a cyclic $\mathbb{Z} \Gamma$-module, $A \simeq \mathbb{Z} \Gamma / I$, where $I$ is a right ideal of $\mathbb{Z} \Gamma$. By Schur's Lemma, $A \otimes_{\mathbb{Z}} \mathbb{Q}$ has a simple $\mathbb{Q} F$-submodule and hence the element $z$ may be chosen such that $I \cap \mathbb{Z} F=R$ is a prime ideal of $\mathbb{Z} F$. Put $B=\mathbb{Z} \Gamma / R \mathbb{Z} \Gamma=K \otimes_{\mathbb{Z} F} \mathbb{Z} \Gamma=\bigoplus_{i=1}^{n} K t_{i}$, where $\left\{t_{1}, \ldots, t_{n}\right\}$ is a right transversal to $F$ in $\Gamma$ and $K \simeq \mathbb{Z} F / R$. Then $A \approx B / X$ where $X=I / R \mathbb{Z} \Gamma$. Putting $X \leq C_{B}(M)$ we may consider $M$ as a $k B$-module. It is easy to check that $\mathscr{A}_{k B}^{+}(x) / X=\mathscr{A}_{k A}^{+}(x)$. Then $\mathscr{A}_{k B}^{+}(x)$ is a dense subgroup of $B$ and hence $\mathscr{A}_{k B}^{+}(x) \cap$ $K t_{i}=J_{i}$ is a dense subgroup in $K t_{i}$ for each $i$. Put $J=\bigcap_{i=1}^{n} J_{i} t_{i}^{-1}$; then $J$ is a dense subgroup in $K$. By Lemma 3.3, there is an endomorphism $g$ of the $\mathbb{Z} F$-module $K$ such that $J$ has a $\mathbb{Z}[g]$-submodule $H$ such that $K / H$ is a Cernikov $\mathbb{Z}[g]$-module with cyclic socle. As $H \leq J \leq J_{i} t_{i}^{-1}$ and $J_{i} \leq \mathscr{A}_{k B}^{+}(x), H t_{i} \leq \mathscr{A}_{k B}^{+}(x)$ for each $i$ and hence $V=\bigoplus_{i=1}^{n} H t_{i} \leq \mathscr{A}_{k B}^{+}(x)$. Thus $V \leq C_{B}(x k B)$ and hence $x k B$ can be considered as a $k(B / V)$-module. Putting 
$b^{g}=\sum_{i=1}^{n} b_{i}^{g} t_{i}$ for any $b=\sum_{i=1}^{n} b_{i} t_{i} \in B$, we can consider $g$ as a $\mathbb{Z} \Gamma$-endomorphism of $B$. Thus, $B$ is a $\mathbb{Z}[g]$-module and $V$ is a submodule of $B$. Then $B / V=\bigoplus_{i=1}^{n}(K / H) t_{i}$, where $(K / H) t_{i}$ is a Cernikov $\mathbb{Z}[g]$-module with cyclic socle for each $i$. So, by Proposition 2.6, there is an element $y \in x k B \backslash\{0\}$ such that $k C_{i} \cap \mathscr{A}_{k B}(y)=P_{i}$ is a maximal ideal of $k C_{i}$, where $C_{i} / D_{i}=\operatorname{Soc}\left(K t_{i} / D_{i}\right)$ and $D_{i}$ is the maximal $g$-invariant subgroup of $\mathscr{A}_{k B}^{+}(y) \cap K t_{i}$. Put $D=\bigoplus_{i=1}^{n} D_{i}$.

Let $\gamma$ be an element of $\Gamma$ which is not contained in $N_{\Gamma}(D)$. It is not difficult to show that, for any $i, D_{i}^{\gamma} \leq K t_{j}$ for some $j$ and hence, as $D^{\gamma}=\bigoplus_{i=1}^{n} D_{i}^{\gamma}$ and $D^{\gamma} \neq D, D_{i}^{\gamma} \leq K t_{j}$ and $D_{i}^{\gamma} \neq D_{j}$ for some $i$ and $j$. As $g$ is a $\mathbb{Z} \Gamma$-endomorphism of $B, D^{\gamma}$ is a $\mathbb{Z}[g]$-submodule of $K t_{j}$ and hence, as $D \gamma \neq D_{j}, L=C_{j} \cap D_{i}^{\gamma} \neq D_{j}$. Since $L \leq C_{j}, k L \cap \mathbb{A}_{k B}(y)=P$ is a maximal ideal of $k L$.

Suppose that $D_{i}^{\gamma}$ is not contained in $D_{j}$. Then, as $D_{j}$ is the maximal $g$-invariant subgroup of $K t_{j} \cap \mathscr{A}_{k B}^{+}(y), \mathscr{A}_{k B}^{+}(y)$ does not contain $L$. Since $L \leq D_{i}^{\gamma}, L \leq\left(\mathscr{A}_{k B}^{+}(y)\right)^{\gamma}$ and, by Lemma 3.4, $\mathrm{Sp}\left(\mathscr{A}_{k B}(y)\right) \cap \operatorname{Sp}\left(\mathscr{A}_{k B}(y)\right)=\varnothing$. If $D_{\gamma}^{\gamma}<D_{j}$ then $D_{j}^{\gamma^{-1}}$ is not contained in $D_{i}$ and the same arguments show that $\operatorname{Sp}\left(\mathscr{A}_{k B}(y)\right) \cap \operatorname{Sp}\left(\mathscr{A}_{k B}^{\gamma^{-1}}(y)\right)=\varnothing$. Therefore, as $\operatorname{Sp}\left(\mathscr{A}_{k B}(y)\right)^{\gamma}=\operatorname{Sp}\left(\mathscr{A}_{k B}(y)\right), \operatorname{Sp}\left(\mathscr{A}_{k B}(y)\right) \cap \operatorname{Sp}\left(\mathscr{A}_{k B}(y)\right)=\varnothing$.

As $X \leq C_{B}(x k B)$ and $y \in x k B, X \leq C_{B}(y k B)$ and hence $X \leq \mathscr{A}_{k B}^{+}(y)$. Since $X$ is a $\Gamma$-invariant subgroup of $B, N_{\Gamma}(D) \leq N_{\Gamma}(X D)=N_{\Gamma}(W)$, where $W=X D / X$, and hence $\operatorname{Sp}\left(\mathscr{A}_{k B}(y)\right) \cap \operatorname{Sp}\left(\mathscr{A}_{k B}^{\gamma}(y)\right)=\varnothing$ if $\gamma$ is not contained in $N_{\mathrm{r}}(W)$. Let $\Delta$ be the ideal of $k B$ generated by $1-X$. Then it is not difficult to show that $\mathscr{A}_{k B}(y) / \Delta=\mathscr{A}_{k A}(y)$ and the theorem follows from Lemma 3.1(ii).

Lemma 3.6. Let $A$ be an abelian torsion-free group of finite rank acted upon by a soluble group $\Gamma$ of finite torsion-free rank and let $K$ be a subgroup of $\Gamma$ such that $r_{0}(K)=r_{0}(\Gamma)$. If $\Delta_{\Gamma}(A)=1$ then $\Delta_{K}(A)=1$.

Proof. Evidently, we may assume that $C_{\mathrm{r}}(A)=1$. Then it easily follows from Lemma 3.2(i) that $\Gamma$ has an abelian normal torsion-free subgroup $H$. The proof is by induction on $r_{0}(\Gamma)$. Suppose that $\Delta_{K}(A) \neq 1$. As $r_{0}(K)=r_{0}(\Gamma), H / V$ is a torsion group, where $V=H \cap K$. Let $1 \neq d \in \Delta_{K}(A)$ and let $D=\left\langle d^{h} \mid h \in H\right\rangle$; it is not difficult to show that $D \leq \Delta_{V}(A)$. Let $B$ be a dense finitely generated subgroup of $D$. As $D \leq \Delta_{V}(A)$, $\left|V: C_{V}(B)\right|<\infty$ and hence $H / C_{H}(D)$ is a torsion-group. Then, since $D$ is an abelian torsion-free group of finite rank, by Lemma 3.2(i), $\left|H / C_{H}(D)\right|<\infty$. Hence there is $n \in \mathbb{N}$ such that $H^{n} \leq C_{H}(D)$. Since $H^{n}$ is a normal subgroup of $\Gamma, C_{A}\left(H^{n}\right)=C$ is a $\Gamma$-invariant subgroup of $A$. Then $r_{0}\left(\Gamma / C_{\Gamma}(C)\right)<r_{0}(\Gamma)$ and hence, by the induction hypothesis, $\Delta_{K}(C)=1$ but this is a contradiction because $D \leq C$.

Lemma 3.7. Let $A$ be an abelian torsion-free group of finite rank acted upon by a group $\Gamma$ such that $\Delta_{\Gamma}(A)=1$. Let $k$ be a field and let $M$ be a $k A$-module. Suppose that there is an element $x \in M$ such that $\mathscr{A}_{k A}(x)$ is a non-zero locally prime ideal of $k A$ and $S_{\Gamma}\left(\mathscr{A}_{k A}(x)\right)=\Gamma$. Then there is a non-trivial $\Gamma$-invariant subgroup $B$ of $A$ such that $B \cap \mathscr{A}_{k A}^{+}(x)$ is a dense subgroup of $B$. 
Proof. By [18, Section 3.11], $\mathscr{A}_{k A}^{+}(x) \neq 1$ and $B$ may be chosen as the isolator of $\mathscr{A}_{k A}^{+}(x)$ in $A$.

THEOREM 3.8. Let $A$ be an abelian torsion-free group of finite rank acted upon by a soluble group $\Gamma$ of finite torsion-free rank such that $\Delta_{\mathrm{r}}(A)=1$. Let $k$ be a field of characteristic zero and let $M$ be a kA-module. Suppose that there is an element $x \in M \backslash\{0\}$ such that $\mathscr{A}_{k A}(x)$ is a non-zero locally prime ideal of $k A$ and $r_{0}\left(\operatorname{Sep}_{r}\left(\mathscr{A}_{k A}(x)\right)\right)=r_{0}(\Gamma)$. Then there is an element $y \in M \backslash\{0\}$ such that $\mathscr{A}_{k A}^{+}(y)$ has a non-trivial $\operatorname{Sep}_{\Gamma}\left(\mathscr{A}_{k A}(y)\right)$ invariant subgroup.

Proof. Put $\operatorname{Sep}_{\Gamma}\left(\mathscr{A}_{k A}(x)\right)=S$. Then, by Lemma 3.6, $\Delta_{S}(A)=1$ and we may assume that $\operatorname{Sep}_{\Gamma}\left(\mathscr{A}_{k A}(x)\right)=\Gamma$. As $S_{\Gamma}\left(\mathscr{A}_{k A}(x)\right) \geqslant \operatorname{Sep}_{\Gamma}\left(\mathscr{A}_{k A}(x)\right), S_{\Gamma}\left(\mathscr{A}_{k A}(x)\right)=\Gamma$. By Lemma 3.7, there is a non-trivial $\Gamma$-invariant subgroup $B$ of $A$ such that $\mathscr{A}_{k A}^{+}(x) \cap B$ is a dense subgroup in $B$. Then the theorem easily follows from Theorem 3.5 and Lemma 3.1(i). rank.

4. A control theorem for modules over group algebras of soluble groups of finite

PROPOSITION 4.1. Let $G$ be a group with abelian normal torsion-free subgroup $A$ of finite rank, and let $B$ be a dense finitely generated subgroup of $A$. Let $k$ be a field and let $M$ be a $k G$-module which is not $k A$-torsion-free. Then:

(i) there is $x \in M \backslash\{0\}$ with the prime annihilator $P_{0}$ in $k B$ such that the transcendence degree of the fraction field of the ring $k B / P_{0}$ is minimal and hence $x$ has maximal annihilator in $k B$;

(ii) if $x$ satisfies (i) then $x k G=x k H \otimes_{k H} k G$, where $H=\operatorname{Sep}_{G}\left(\mathscr{A}_{k A}(x)\right)$.

Proof. (i) This assertion is proved in [12, Theorem E].

(ii) By [12, Theorem E] there is a prime ideal $P$ of $k A$ such that $P \cap k B=P_{0}$ and $x k G=x k S \otimes_{k S} k G$, where $S=S_{G}(P)$. As $P \cap k B=P_{0}=\mathscr{A}_{k B}(x)$, it is not difficult to show that $S=S_{G}\left(\mathscr{A}_{k A}(x)\right)$. Thus, it is sufficient to show that $x k S=x k H \otimes_{k H} k S$, where $H=\operatorname{Sep}_{G}\left(\mathscr{A}_{k A}(x)\right)$. So, we may assume that $S=G$.

Put $J=\mathscr{A}_{k G}(x)$; then it is sufficient to show that $J=(J \cap k H) k G$. Suppose that $J \neq(J \cap k H) k G$; then there is an element $q \in J$ which is not belonging to $(J \cap k H) k G$. Put $q=\sum_{i=1}^{n}\left(\sum_{j=1}^{k_{i}} \alpha_{i j} d_{i j}\right) t_{i}$, where $\alpha_{i j} \in k A,\left\{d_{i j}\right\}$ is a part of a right transversal to $A$ in $H$ and $\left\{t_{i}\right\}$ is a part of a right transversal to $H$ in $G$. The element $q$ can be chosen such that $m=\sum_{i=1}^{n} k_{i}$ is minimal with respect $q \in J$ and $q$ is not contained in $(J \cap k H) k G$. We can also assume that $t_{1}=e$ and $d_{11}=e$. Put $g_{i j}=d_{i j} t_{i}$ then for any $g_{i j}$ and any $\beta \in I$ the element $q \beta^{g_{i j}}$ can be written in the form: $q \beta^{g_{i j}}=\sum_{l=1}^{n}\left(\sum_{r=1}^{k_{l}} \hat{\alpha}_{l r} d_{l r}\right) t_{l}$, where $\hat{\alpha}_{l r}=\alpha_{l r} \beta^{h_{b}} \in k A$ and $h_{l r}=g_{i j} g_{l r}^{-1}$. Therefore, as $\alpha_{i j} g_{i j} \beta^{g_{i i}}=\beta \alpha_{i j} g_{i j} \in(J \cap k H) k G, b=q \beta^{g_{i i}}-\alpha_{i j} g_{i j} \beta^{g_{i j}} \in J$ for any $\beta \in I$. As the number of summands in $b$ less than $m$, it follows from minimality of $m$ that $b \in(J \cap k H) k G$ and hence, as $\alpha_{i j} g_{i j} \beta^{g_{i j}} \in(J \cap k H) k G, q \beta^{g_{i j}} \in(J \cap k H) k G$. Therefore, as $t_{1}=e, \sum_{r=1}^{k_{1}} \hat{\alpha}_{1 r} d_{1 r}=\left(\sum_{r=1}^{k_{1}} \alpha_{1 r} d_{1 r}\right) \beta^{g_{i j}} \in J \cap k H$. Put $c=\sum_{r=1}^{k_{1}} \alpha_{1 r} d_{1 r} ;$ then $c \beta^{g_{i j}} \in J$ for any $\beta \in I$ and hence $c I^{g_{i j}} \subseteq J$. Thus, $I^{g_{i i}} \subseteq \mathscr{A}_{k A}(y)$, for any $g_{i j}$, where $y=x c$. Then, as $g_{11}=e$, it is not 
difficult to show that $\operatorname{Sp}(I) \cap \operatorname{Sp}\left(I^{g_{i i}}\right) \neq \varnothing$ for any $g_{i j} \neq e$. This is a contradiction, because $g_{i j} \neq e$ is not contained in $H$ and $H$ separates $I$.

THEOREM 4.2. Let $G$ be a soluble group of finite torsion-free rank and let $A$ be $a$ torsion-free abelian normal subgroup of $G$ such that $\Delta_{G}(A)=1$. Let $k$ be a field of characteristic zero and let $M$ be a $k G$-module. If $M$ is not $k A$-torsion-free then there is an element $a \in M \backslash\{0\}$ such that $a k G=(a k H) \oplus_{k H} k G$ and $r_{0}\left(H / C_{H}(a k H)\right)<r_{0}(G)$, where $H=\operatorname{Sep}_{G}\left(\mathscr{A}_{k A}(a)\right.$.

Proof. Let $B$ be a finitely generated dense subgroup of $A$. By Proposition 4.1(i), there is an element $x \in M \backslash\{0\}$ such that $\mathscr{A}_{k B}(x)$ is a prime ideal of $k B$ and the transcendence degree of the fraction field of the ring $k B / \mathscr{A}_{k B}(x)$ is minimal, and hence $x$ has maximal annihilator in $k B$.

Put $K=\operatorname{Sep}_{G}\left(\mathscr{A}_{k A}(x)\right)$. Then by Proposition 4.1(ii), $x k G=x k K \otimes_{k K} k G$. If $r_{0}(K)<$ $r_{0}(G)$ then we may put $a=x$ and $H=K$.

Suppose that $r_{0}(K)=r_{0}(G)$. Evidently, $\mathscr{A}_{k A}(x)$ is a locally prime ideal of $k A$. Then, by Theorem 3.8, there is an element $a \in x K A \backslash\{0\}$ such that $\mathscr{A}_{k A}^{+}(a)$ has a non-trivial $H$-invariant subgroup $D$, where $H=\operatorname{Sep}_{G}\left(\mathscr{A}_{k A}(a)\right)$. Therefore, $D \leq C_{H}(a k H)$ and hence $r_{0}\left(H / C_{H}(a k H)\right)<r_{0}(G)$. As $a \in x K A, \mathscr{A}_{k B}(a) \geq \mathscr{A}_{k B}(x)$, and hence, as $x$ has maximal annihilator in $k B, \mathscr{A}_{k B}(a)=\mathscr{A}_{k B}(x)$. Thus, the theorem follows from Proposition 4.1(ii).

\section{An application.}

LEMMA 5.1. Let $G$ be a metabelian finitely generated group of finite Prüfer rank and let $B$ be the derived subgroup of $G$. If the group $G$ is not nilpotent-by-finite then there is $a$ normal subgroup $A$ of $G$ such that $A \leq B, \Delta_{G}(A)=1$ and the quotient group $G / A$ is nilpotent-by-finite.

Proof. The proof is by induction on $r_{0}(B)$. Let $T$ be the torsion subgroup of $B$. As $G$ has the maximal condition for normal subgroups (see [5]) and Prufer rank of $T$ is finite, $T$ is finite. If $T=B$ then the group $G$ is abelian-by-finite. Thus we may assume that $T \neq B$. Then there is $n \in \mathbb{N}$ such that $C=B^{n}$ is a torsion-free subgroup. If $\Delta_{G}(C)=1$ then we can put $A=C$. Thus we may assume that $\Delta_{G}(C) \neq 1$. Put $D=\left\langle d^{g} \mid g \in G\right\rangle$, where $d$ is a non-identity element of $\Delta_{G}(C)$. It is easy to check that if the quotient group $G / D$ is nilpotent-by-finite then so is $G$. Therefore, $G / D$ is not nilpotent-by-finite and hence, by the induction hypothesis, there is a normal subgroup $E$ of $G$ such that $D \leq E \leq B$ and $\Delta_{G}(E / D)=1$. We will consider $E$ as a $\mathbb{Z} \Gamma$-module, where $\Gamma=G / C_{G}(E)$. Since $E \leq B, \Gamma$ is an abelian group. Evidently, the subgroup $E$ may be chosen such that $(E / D) \otimes_{\mathbb{Z}} \mathbb{Q}$ is a simple $\mathbb{Q} \Gamma$-module. Since $d \in \Delta_{G}(C),\left|G: C_{\Gamma}(D)\right|<\infty$ and hence, as $\Delta_{\Gamma}(E / D)=1$, there is an element $\gamma \in C_{\Gamma}(D)$ which is not contained in $C_{\Gamma}(E)$. Then, as the group $\Gamma$ is abelian, the mapping $\varphi$ given by $\varphi: x \rightarrow x(1-\gamma)$ is a non-zero $\mathbb{Z} \Gamma$-endomorphism of $E$ such that $D \leq \operatorname{Ker} \varphi$. Hence, as $(E / D) \otimes_{\mathbb{Z}} \mathbb{Q} \Gamma$ is a simple $\mathbb{Q} \Gamma$-module, $\operatorname{Ker} \varphi=D$. Then $L=\varphi(E) \simeq$ $E / D$ and hence $\Delta_{\Gamma}(L)=1$. Thus, $L$ is a normal subgroup of $G$ such that $L \leq B$ and $\Delta_{G}(L)=1$. So, passing to the quotient group $G / L$ we can use the induction hypothesis.

LemMA 5.2. Let $S$ be a commutative ring acted upon by a group $G$, let $M$ be an 
$S$-module and let $F$ be a submodule of $M$. Suppose that there is a non-zero element $\alpha \in S$ such that each element of $M / F$ is annihilated by some product $\alpha^{B_{1}} \ldots \alpha^{g_{k}}$ of conjugates of $\alpha$ by elements of $G$. Then for any non-zero ideal $L$ of $S$ each element of $(M L \cap F) / F L$ is annihilated by some product $\alpha^{g_{1}} \ldots \alpha^{g_{k}}$ of conjugates of $\alpha$ by elements of $G$.

Proof. Each element $a \in M L \cap F$ can be written in the form: $a=\sum_{i=1}^{n} a_{i} l_{i}$, where $a_{i} \in M$ and $l_{i} \in L$. Then there is an element $x=\alpha^{g_{1}} \ldots \alpha^{g_{k}}$ where $g_{i} \in G$ such that $a_{i} x \in F$ for each $i$ and hence $a x=\sum_{i=1}^{n} a_{i} x l_{i} \in F L$.

LEMMA 5.3. Let $A$ be a torsion-free abelian minimax group acted upon by an abelian group $\Gamma$ such that $A \otimes_{\mathbb{Z}} \mathbb{Q}$ is a simple $\mathbb{Q} \Gamma$-module. Let $k$ be a field of characteristic zero and let $\alpha$ be a non-zero element of $k A$. Then there is a maximal ideal $L$ of $k A$ such that $\left|A: L^{+}\right|<\infty$ and $L$ contains no conjugates of $\alpha$ by elements of $\Gamma$.

Proof. Put $\alpha=\sum_{i=1}^{n} \alpha_{i} t_{i}$, where $\alpha_{i} \in k$ and $t_{i} \in A$, and let $F$ be a subfield of $k$ generated by $\alpha_{i}$; then $\alpha \in F A$. Let $\mathscr{L}$ be the set of $\Gamma$-invariant maximal ideals $M$ of $F A$ with $\left|A: M^{+}\right|<\infty$; then, by $[\mathbf{1 7}$, Theorem A], the intersection of ideals from $\mathscr{L}$ is zero. It easily implies that there is $M \in \mathscr{L}$ which contains no conjugates of $\alpha$ by elements of $\Gamma$. Then $L$ may be chosen as a maximal ideal of $k A$ which contains $M$.

Lemma 5.4. Let $G$ be a finitely generated metabelian group of finite Prüfer rank, let $k$ be a field and let $M$ be a simple $k G$-module. Let $A$ be an abelian torsion-free normal subgroup of $G$ such that $A$ is contained in the derived subgroup of $G$ and the quotient group $G / A$ is polycyclic. Then the module $M$ is not $k A$-torsion-free.

Proof. By [4, Corollary 2.1], there are a free $k A$-submodule $F$ of $M$ and a non-zero element $\alpha \in k A$ such that each element of $M / F$ is annihilated by some product $\alpha^{g_{1}} \ldots \alpha^{g_{k}}$ of conjugates of $\alpha$ by elements of $G$. Let $C$ be a normal subgroup of $G$ such that $C \leq A$, the quotient group $A / C$ is torsion-free and $C \otimes_{\mathbb{Z}} \mathbb{Q}$ is a simple $\mathbb{Q} G$-module. Then the element $\alpha$ may be written in the form $\alpha=\sum_{i=1}^{n} \alpha_{i} t_{i}$, where $\alpha_{i} \in k C$ and $\left\{t_{1}, \ldots, t_{n}\right\}$ is a part of a transversal to $C$ in $A$. Put $\beta=\prod_{i=1}^{n} \alpha_{i}$; as $k C$ has no zero divisors, $\beta \neq 0$. By [6, Lemma 5.1], the subgroup $C$ is minimax and hence, as the quotient group $\Gamma=G / C_{G}(A)$ is abelian there is, by Lemma 5.3, a maximal ideal $L$ of $k C$ such that $\left|C: L^{+}\right|<\infty$ and $L$ contains no conjugates of $\beta$ by elements of $G$. It implies that $k A L$ contains no conjugates of $\alpha$ by elements of $G$.

Since $\left|C: L^{+}\right|<\infty$, it is not difficult to show that $L$ contains a non-zero $G$-invariant ideal $I$. As the ideal $I$ is $G$-invariant, it is not difficult to show that $M I$ is a submodule of $M$ and hence, as the module $M$ is simple, either $M I=0$ or $M I=M$. If $M I=0$ then the lemma holds. Thus we may assume that $M I=M$ and hence $M L=M$. Then, by Lemma 5.2 , each element of $F / F L$ is annihilated by some product $\alpha^{g_{1}} \ldots \alpha^{g_{k}}$ of conjugates of $\alpha$ by elements of $G$. As $F$ is a free $k A$-module, $\oplus_{i}(k A / k A L)_{i}=F / F L$ and hence some such product $\alpha^{g_{1}} \ldots \alpha^{g_{k}}$ is contained in $k A L$. It is not difficult to note that the quotient ring 
$k A / k a L$ may be considered as a crossed product (see [13]) of a field $k C / L$ and the torsion-free quotient group $A / C$. It is well known that such a crossed product has no zero divisors and hence, as $k A L$ contains no conjugates of $\alpha$ by elements of $G, \alpha^{g_{1}} \ldots \alpha^{g_{k}}$ may not be contained in $k A L$. This is a contradiction.

THEOREM 5.5. Let $G$ be a finitely generated metabelian group of finite Prüfer rank, let $k$ be a field of characteristic zero and let $M$ be an irreducible $k G$-module such that $C_{G}(M)=1$. If the group $G$ is not nilpotent-by-finite then there are a subgroup $H \leq G$ and an irreducible $k H$-submodule $U \leq M$ such that $M=U \otimes_{k H} k G$ and $r_{0}(H)<r_{0}(G)$.

Proof. The proof is by induction on $r_{0}(G)$. By Lemma 5.1, there is an abelian normal torsion-free subgroup $A \leq G$ such that $\Delta_{G}(A)=1$ and the quotient group $G / A$ is nilpotent-by-finite. As the group $G$ is finitely generated, the quotient group $G / A$ is polycyclic. Then, by Lemma 5.4, $M$ is not $k A$-torsion-free. By Theorem 4.2, there is an element $a \in M$ such that $M=U \otimes_{k H} k G$ and $r_{0}\left(H / C_{H}(U)\right)<r_{0}(G)$, where $U=a k A$ and $H=\operatorname{Sep}_{G}\left(\mathscr{A}_{k A}(a)\right)$. Evidently, $H$ contains the derived subgroup of $G$ and hence, as the quotient group $G / A$ is polycyclic, if $|G: H|=\infty$ then $r_{0}(H)<r_{0}(G)$. Thus we may assume that $|G: H|<\infty$. Since $H$ contains the derived subgroup of $G, H$ is a normal subgroup of $G$. Then $H$ is a finitely generated subgroup. Suppose that the quotient group $H / C_{H}(U)$ is nilpotent-by-finite. Let $\left\{t_{1}, \ldots, t_{m}\right\}$ be a right transversal to $H$ in $G$. As $M=\oplus_{i=1}^{m} U t_{i}$, $C_{G}(M) \geq \bigcap_{i=1}^{m}\left(C_{H}(U)\right)^{t_{i}}=C$ and therefore, as $C_{G}(M)=1, C=1$. Then, by Remak's theorem, $\prod_{i=1}^{m}\left(H / C_{H}(U)^{i_{i}}\right) \leq H$. It easily follows that the subgroup $H$ is nilpotent-by-finite and hence, as $|G: H|<\infty$, so is $G$, and a contradiction ensues. Thus, the quotient group $H / C_{H}(U)$ is not nilpotent-by-finite and we may use the induction hypothesis.

COROLlary 5.6. Let $G$ be a finitely generated group of finite Prüfer rank, and let $k$ be a field of characteristic zero. Suppose that $G$ is an extension of an abelian group $A$ by a cyclic group $\langle g\rangle$. If the group $G$ is not nilpotent-by-finite then every faithful irreducible representation of $G$ over $k$ is induced from an irreducible representation of the group $A$ over $k$.

Proof. It is not difficult to note that the subgroup $H$ in the proof of Theorem 5.4 contains $A$. As $r_{0}(H)<r_{0}(G)$, it implies that $A=H$.

ACKNowledgement. I am deeply grateful to the referee for his very helpful comments and advice.

\section{REFERENCES}

1. C. J. B. Brookes, Ideals in group rings of soluble groups of finite rank, Math. Proc. Camb. Phil. Soc. 97 (1985), 27-49.

2. C. J. B. Brookes and K. A. Brown, Primitive group rings and Noetherian rings of quotients. Trans. Amer. Math. Soc. 288 (1985), 605-623.

3. C. J. B. Brookes and K. A. Brown, Injective modules, induction maps and endomorphism rings. Proc. London Math. Soc. (3) 67 (1993), 127-158. 
4. K. A. Brown, The Nullstellensatz for certain group algebras. J. London Math. Soc. 26 (1982) 425-434. 419-436.

5. P. Hall, Finiteness conditions for soluble groups. Proc. London Math. Soc. 4 (1954) $595-622$.

6. P. Hall, On the finiteness of certain soluble groups. Proc. London Math. Soc. 9 (1959)

7. D. L. Harper, Primitive irreducible representation of nilpotent groups. Math. Proc. Camb. Phil. Soc. 82 (1977), 241-247.

8. D. L. Harper, Primitivity in representations of polycyclic groups. Math. Proc. Camb. Phil. Soc. 88 (1980), 15-31.

9. B. Hartley, A dual approach to Cernikov modules. Math. Proc. Camb. Math. Soc. 82 (1977), 215-239. 43-52:

10. I. M. Musson, Representations of infinite soluble groups. Glasgow Math. J. 24 (1983),

11. I. M. Musson, Irreducible modules for polycyclic group algebras. Canad. J. Math. 33 (1981), 901-914.

12. I. T. Nabney, Soluble minimax groups and their representations. Ph.D. thesis (University of Cambridge, 1989).

13. D. S. Passman, Infinite crossed products (Academic Press, Boston, 1989).

14. J. E. Roseblade, Group rings of polycyclic groups, J. Pure Appl. Algebra. 3 (1973) 307-328.

15. D. Segal, Irreducible representations of finitely generated nilpotent groups, Math. Proc. Camb. Phil. Soc. 81 (1977), 201-208.

16. B. A. F. Wehrfritz, Infinite linear groups (Springer-Verlag, 1973).

17. B. A. F. Wehrfritz, Invariant maximal ideals in certain group algebras. J. London Math. Soc. 46 (1992), 101-110.

18. J. S. Wilson, Soluble products of minimax groups, and nearly surjective derivations. $J$. Pure and Appl. Algebra. 53 (1988), 297-318.

Department of Mathematics

UNIVERSITY OF DNEPROPETROVSK

Prospect Gagarina 72

DNEPROPETROVSK, 320625

UKRAINE 